\title{
Conductance statistics in small GaAs:Si wires at low temperatures. I. Theoretical analysis: truncated quantum fluctuations in insulating wires
}

\author{
François Ladieu and Jean-Philippe Bouchaud \\ Service de Physique de l'Etat Condensé, CEA-Saclay, 91191 Gif/Yvette Cedex, France \\ (Received 20 April 1993, received in final form 5 July 1993, accepted 13 July 1993)
}

\begin{abstract}
Résumé. - Nous analysons en détail le régime de saut à portée variable de Mott à une dimension, allant au delà d'un travail précédent dû à Raihk et Ruzin. Nous montrons que les très grandes fluctuations de conductance dans les isolants désordonnés résultent d'une combinaison subtile entre des phénomènes purement quantiques et des fluctuations géométriques dues aux positions et aux énergies des impuretés. Nos résultats se comparent très favorablement à la fois à des résultats expérimentaux et à des simulations numériques.
\end{abstract}

\begin{abstract}
We analyse in detail Mott's variable range hopping in one dimension, expanding on earlier work by Raikh and Ruzin. We show that the large conductance fluctuations in disordered insulators result from a subtle interplay between purely quantum phenomena and geometrical fluctuations arising from the energies and locations of the impurities. Our results compare very well with both experiments and numerical simulations.
\end{abstract}

In recent years, theoretical and experimental works have underlined the importance of conductance fluctuations in disordered conductors [1]. While the situation is rather well understood in the metallic phase, where "universal conductance fluctuations" $\delta g \simeq \frac{e^{2}}{\hbar}$ are observed, less is known in the strongly localized regime. On the experimental side, several groups have observed reproducible conductance fluctuations on quasi one dimensional (insulating) wires [2, 3]. These fluctuations have been argued to be of quantum origin [4]: the transmission coefficient of a disordered bar is known to depend sensitively on the energy (i.e. the gate voltage in the experiments) and on the configuration of the impurities. The size of these (zero temperature) fluctuations have been computed within the random matrix theory, [5] and the result is that var $\ln g=-\langle\ln g\rangle \equiv L / \xi$, where $L$ is the length of the sample and $\xi$ the localisation length. Another theory, based on the "directed path approximation" predicts somewhat smaller fluctuations [6] : var $\ln g \propto C L^{2 \omega}$, where $\omega$ is an exponent close to $1 / 5$ (in three dimensions) and 
$C$ a prefactor independent of $\langle\ln g>$ : in other words, in this theory var $\ln g$ and $\langle\ln g>$ may evolve differently with e.g. magnetic field or gate voltage. For non-zero temperatures, however, the inelastic collision time is much shorter than the time needed for the electron to cross the sample, and activated energy jumps between impurities come into play: one enters Mott's variable range hopping regime $[7,8]$, where phase coherence is only preserved at length scales shorter than the typical jump size $r_{0}$. Lee [9] has argued, on the basis of numerical simulations (see also [10)], that the "geometrical fluctuations" in the location and energies of the impurities were sufficient, in the one-dimensional Mott regime, to induce large, reproducible conductance fluctuations. These fluctuations would thus be unrelated to the quantum, i.e. interference, effects depicted in [4] which have been shown to be relevant only at very low temperature [11]. Comparison with the experiments, however, show that the size of the fluctuations obtained in reference [9] is too large [3].

The aim of the article is twofold. We first show how to solve analytically Mott's problem in one dimension. This provides a clear interpretation of the conductance of a finite disordered wire, which is limited by the "weakest link" in the sample, and allows us to characterize the fluctuations and discuss their dependence on the length of the sample. These results are however almost identical to those of Raikh and Ruzin [12] - although these authors relied on a simplified analysis. As already discussed [12], one reaches quantitative agreement with the numerical data $[9,10]$. To be able to describe all the features of the experiments, we then show that quantum fluctuations governing the fluctuations of the weakest link's conductivity must be taken into account. However, due to the presence of other links, these fluctuations cannot develop fully - they are "truncated" by geometrical effects. Details concerning the experiments are published in the companion paper [3].

We thus start with Mott's problem in one dimension. We assume that the energy levels are independent and randomly scattered with a density of states $\rho$ per unit length and energy. The resistance between two sites $\left(x_{\imath}, E_{\imath}\right),\left(x_{\jmath}, E_{\jmath}\right)$ is given by (see [13])

$$
R_{\imath \jmath}=R^{*} \exp \left[\frac{\left|E_{\imath}\right|+\left|E_{\jmath}\right|+\left|E_{\imath}-E_{\jmath}\right|}{2 k T}+\frac{\left|r_{\imath}-r_{\jmath}\right|}{\xi}\right]
$$

where $R^{*}$ is a typical (metallic) resistivity at scale $\xi$, which will be set to 1 in the following, and $E=0$ corresponds to the Fermi level. Note that equation (1) completely neglects the fluctuations of quantum origin, which can be seen as giving a (Gaussian) distribution to $1 / \xi$ [5]. These quantum fluctuations will be considered later.

The problem is thus to determine from equation (1) the total resistance between $(x=$ $0, E=0$ ) and ( $x=L, E=0$ ) of a given sample, knowing the distribution of $\left(x_{2}, E_{2}\right)$. Even though the problem is one-dimensional, this is not trivial since each site is connected to every other site. As usual for this problem [13], in view of the exponentially fast variation of $R_{\imath \jmath}$, we approximate the end-to-end resistance with that of the less resistive path. Furthermore, equation (1) shows that the "optimal" path cannot wander arbitrarily far from the Fermi level. We shall thus make the assumption that the optimal path can be constructed by always choosing locally the least resistive link. We will discuss later the validity of this assumption and show that it leads to the exact asymptotic result. [It is, in any case, an upper bound for the resistance]. Now, let us call $P(n \mid m)$ the probability that, at a given point of the optimal path, the electron makes a hop of resistance $R \equiv e^{n}$, having started from a site of energy $E \equiv m k T$. In order to calculate $P(n \mid m)$ let us first answer the following question: what is the probability $\int_{n}^{\infty} \mathrm{d} n P(n \mid m)$ that the least resistive link from this site of energy $m k T$ has a resistance greater or equal to $R \equiv e^{n}$ ? For this to occur, no energy level must be found 
in the hatched area shown in figure 1. This occurs with probability $\left({ }^{1}\right) \propto \exp \left[-\frac{n(n-m)}{n_{0}^{2}}\right]$, where $n_{0}^{2} \equiv \frac{1}{\rho k T \xi}$. $\exp n_{0}$ is thus the usual Mott resistance, and $r_{0} \equiv n_{0} \frac{\xi}{2}$ is the typical jump size (which we assume to be much smaller than the sample length: we neglect the possibility of resonant tunnelling). The total probability to find a given value of $n \equiv \ln R$ is thus $\mathcal{P}(n)=\int_{0}^{n} \mathrm{~d} m P(n \mid m) \mathcal{Q}(m)=\int_{0}^{n} \frac{\mathrm{d} m(2 n-m)}{n_{0}^{2}} \exp \left[-\frac{n(n-m)}{n_{0}^{2}}\right] \mathcal{Q}(m) . \quad \mathcal{Q}$ is the probability to find the electron at energy $m k T$ along the best conducting path. [Note that it is not given by the Boltzmann weight !]. A closed equation for $\mathcal{P}$ can be obtained by noticing that $n$ can only increase when $m$ increases, and hence $\int_{n}^{\infty} \mathrm{d} n^{\prime} P\left(n^{\prime} \mid m\right)=\int_{0}^{m} \mathrm{~d} m^{\prime} P\left(m^{\prime} \mid n\right) .(P(m \mid n)$ is the probability to find the electron at energy $m$ after crossing a resistance $\left.e^{n}\right)$. From this we obtain our central result for $\mathcal{P}$ :

$$
\mathcal{P}(n)=\int_{0}^{n} \frac{\mathrm{d} m(2 n-m)}{n_{0}^{2}} \exp \left[-\frac{n(n-m)}{n_{0}^{2}}\right] \int_{m}^{\infty} \frac{\mathrm{d} n^{\prime} n^{\prime}}{n_{0}^{2}} \exp \left[-\frac{n^{\prime}\left(n^{\prime}-m\right)}{n_{0}^{2}}\right] \mathcal{P}\left(n^{\prime}\right)
$$

This equation has been solved numerically: we show in figure 1 (insert) $\mathcal{P}$ versus the rescaled variable $y=n / n_{0}$. One may show in particular that $P(y) \simeq y^{2}$ for small $y$, and $\mathcal{P}(y) \simeq$ $a \exp \left[-\frac{y^{2}}{2}\right](a \sim 2)$ for large $y\left({ }^{2}\right)$. Note that -due to the self-consistent expression of $\mathcal{Q}(m), \mathcal{P}(n)$ - equation (2) takes explicitly into account the correlations existing between consecutive hops of the optimal path through the value $m$ of the intermediate energy level. These correlations are neglected by Raikh and Ruzin [12]. Thus, comparison between both theories will enlighten on the importance of correlations of hops.

Before going further in our analysis, let us note that our hypothesis of neglecting quantum fluctuations is only valid when the width of their distribution $w_{\mathrm{q}}$ is small enough compared to the width $w_{\text {geo }}$ of geometrical distribution $\mathcal{P}$ studied here. As shown by equation (2), one has $w_{\text {geo }} \simeq n_{0}$, whereas one may estimate that $w_{\mathrm{q}} \simeq n_{0}^{\omega}$, with $\omega=1 / 2$ in the barely insulating regime and $\omega \sim 1 / 5$ in the strongly localised regime. Therefore, our model will be valid when $n_{0}>1$, i.e. it will not hold very close to Anderson's transition.

The end-to-end resistance is then given by the following sum $\mathcal{R}=\sum_{\imath=0}^{N} R_{2}$, where $N=$ $\frac{L}{r_{0}}=\frac{2 L}{n_{0} \xi}$ is the total number of jumps, and $\ln R_{\imath}$ are distributed according to $\mathcal{P}$. The simple addition of resistances amounts to neglecting resonant tunnelling which is justified when $N$ is large. Let us now distinguish two cases:

i) Very long wires. In this case, the usual central limit theorem applies and one finds that the resistance is equal to $N \int_{0}^{\infty} \mathrm{d} n \mathcal{P}(n) e^{n}$. From this, we find that $\ln \overline{\mathcal{R}} \simeq 1.5 n_{0}$ for $n_{0} \sim 1$ (Mott's law), but that due to the slowly decreasing tail of $\mathcal{P}, \ln \overline{\mathcal{R}} \simeq \frac{n_{0}^{2}}{2} \propto T^{-1}$ for $n_{0} \gg 1$. This result was first obtained by Kurkijarvi [15] and then by Raikh and Ruzin [12]. Note that this value of resistance is roughly the largest possible one in a wire of given $T_{0}$ at a given $T$ (since $y<n_{0}$ ) and that occurrence of such hops is unavoidable provided that the wire is long [14].

$\left({ }^{1}\right)$ We neglect Wigner correlations between the energy levels, which is justified as soon as $e^{n} \gg n_{0}^{2}$

$\left({ }^{2}\right)$ Although the whole calculation only makes sense if $y<n_{0}$, beyond which $P(y) \equiv 0$. 


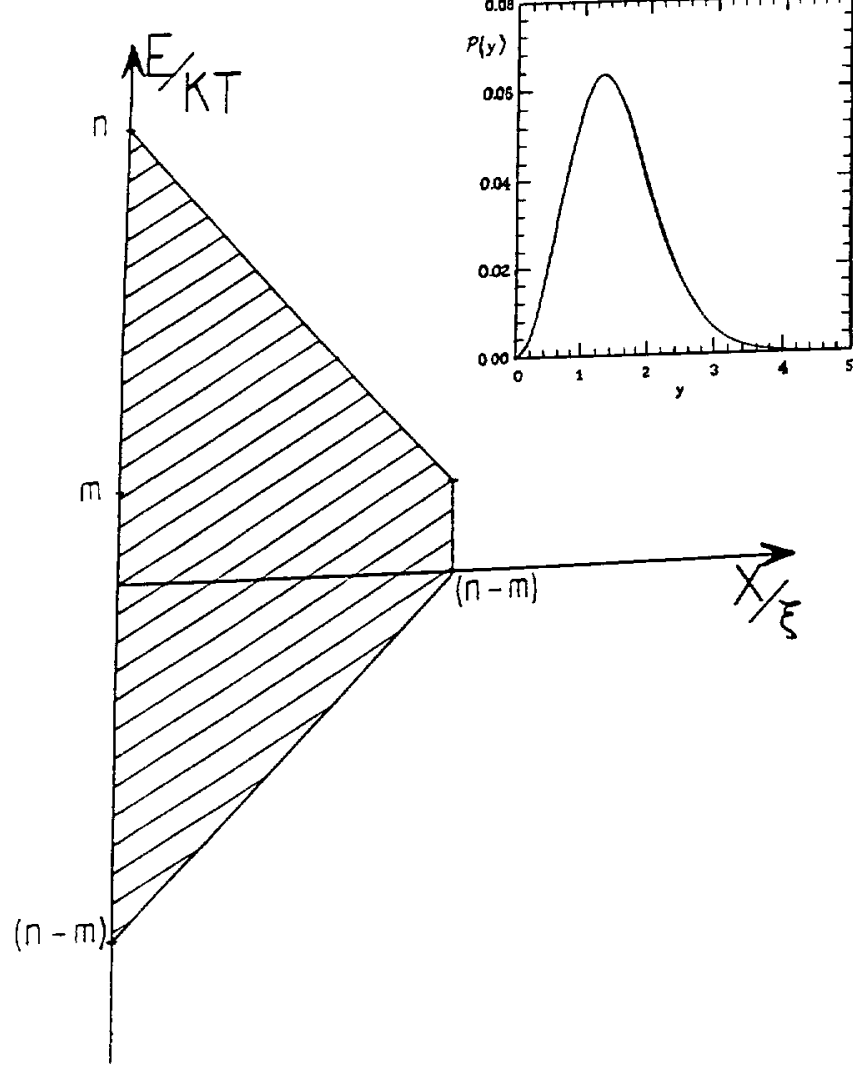

Fig. 1. - Starting from a site with energy $m k T$, no sites must be present in the hatched region of the $(x, E)$ plane for the resistance to be larger than $e^{n}$. Insert: Numerical determination of $\mathcal{P}(y)$ versus $y=\frac{n}{n_{0}}$. The most probable value of $y$ is 1.3 , and the average of $y$ is 1.5 .

enough. Agreement of our result with [12] is thus not surprising since for such "extremely resistive hops" correlations are obviously irrelevant.

ii) Finite wires with $n_{0} \gg 1$. One should however notice that the largest $n$ drawn from $\mathcal{P}$ in $N$ trials is typically of order $n_{\max } \simeq n_{0} \sqrt{2 \ln (a N)}$. The central limit theorem certainly does not apply as long as $n_{\max }<\ln \overline{\mathcal{R}}$ (the mean cannot be larger than the largest element!). In this case, the end-to-end resistance is entirely governed by the weakest link, i.e., by $n_{\max }$. This can also be understood by noting that for intermediate values of $R=\mathrm{e}^{n}$, the distribution $\mathcal{P}(R)$ behaves as a power law $\mathcal{P}(R) \simeq R^{-(1+\mu)}$, with an effective exponent $\mu=\frac{\ln R}{2 n_{0}^{2}}$. For $\mu<1$, the sum giving $\mathcal{R}$ is a "Lévy sum" which is well-known to be given by its few largest terms [16]. From the above results, one may see that $\mu_{\max }=1$ coincides with the condition $n_{\max } \simeq 2 n_{0}^{2}$ : the "anomalous", weak link dominated regime thus prevails for samples shorter than $N^{*} \simeq \frac{1}{a} e^{2 n_{0}^{2}}$, while for longer wires, one reaches the self-averaging regime.

Experimentally [3], $n_{0}$ is in the range $2-5, L \simeq 5 \mu \mathrm{m}, \xi \simeq 20-50 \mathrm{~nm}$, and thus $N$ is between 


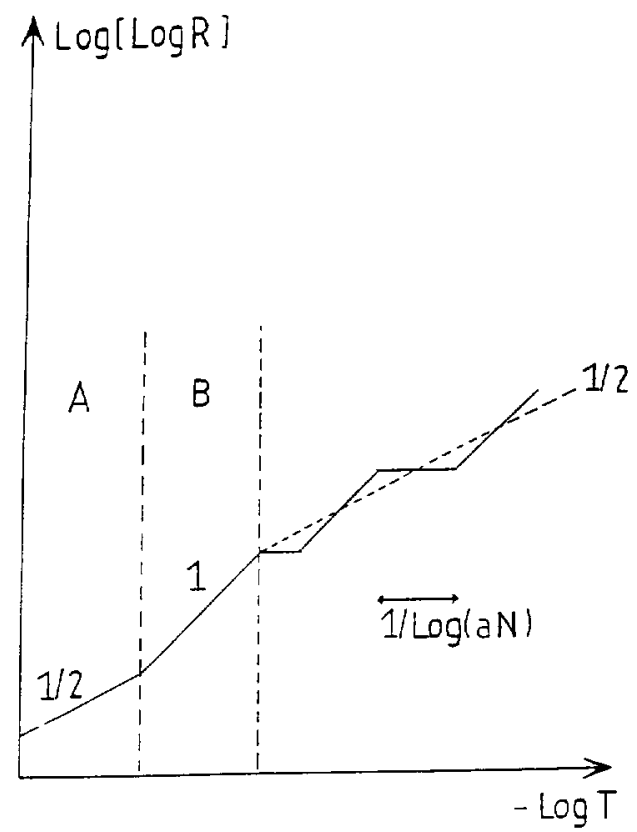

Fig. 2. - Evolution of $\ln \ln \mathcal{R}$ with $-\ln T$ (schematic). For sufficiently long samples, one should see three regions: A: Mott, with slope $1 / 2$, B: Self-averaging regime, with slope 1 , and finally weak link dominated region, with a succession of plateaus (slope 0 ) and activated (slope 1) regions, around an effective Mott law (dotted line).

25 and 100, which is smaller than $N^{*}$ at low temperatures $(T<1 \mathrm{~K})\left({ }^{3}\right)$. In the numerical simulations of Lee [9], $n_{0}^{2}$ varied between 5 and 40 , and $N$ between 12 and 143, again smaller than $N^{*}$. Definite theoretical predictions in this regime are thus of great interest. We have seen that the resistance of the sample is given by $\ln \mathcal{R} \simeq n_{\max }$. The average over disorder $\overline{\ln \mathcal{R}}$ is thus given by:

$$
\bar{n}_{\max } \simeq n_{0} \sqrt{2 \ln (a N)} .
$$

We then conclude that for a given $N<N^{*}, \overline{\ln \mathcal{R}}$ behaves as $n_{0} \propto T^{-1 / 2}$, i.e as Mott would predict, but that the slope of $\overline{\ln \mathcal{R}}$ versus $T^{-1 / 2}$ increases with $N$ as $\sqrt{2 \ln (a N)}$. Let us now turn to the fluctuations: their order of magnitude is governed by the width of the distribution of $n_{\max }$, which can be shown (using the asymptotic shape of $\mathcal{P}$ ) to be $\simeq \frac{n_{0}^{2}}{n_{\max }}$ One then finds:

$$
\frac{\Delta \ln \mathcal{R}}{\ln \mathcal{R}} \simeq \frac{1}{2 \ln a N}
$$

The full distribution of $\ln \mathcal{R}$ was investigated numerically in [10] and analytically in [12]. Equation (3a) and (3b) are in excellent agreement with [10],[12]. However a detailed comparison with [12] reveals a slight change in the length dependence: while here $a$ is a constant $(a \simeq 2)$, in [12] $a=\sqrt{\ln \frac{L}{\xi}}$. Qualitatively, this means that for a given $L$, neglecting correlations leads to a

$\left({ }^{3}\right)$ A numerical calculation shows that $\frac{n_{\max }}{n_{0}}$ varies between 2.1 for $N=5$ to 3.2 for $N=100$ and 3.8 for $N=1000$. 
small increase of $\overline{\ln \mathcal{R}}$. In other words, self-consistent expression of $\mathcal{Q}$ enhances the importance of hops between levels close to the Fermi energy. Equation (1) shows that this leads to a decrease of $\overline{\ln \mathcal{R}}$. However this effect is too small (it goes as $\ln \sqrt{\ln L}$ ) to be numerically tested.

It is noteworthy that Lee used a "percolation" method to obtain the best global conducting path, i.e. the assumption of local optimization is not made. Nevertheless, his numerical results [9] show all the features reported above, in particular equations (3a) and (3b). However, if a very detailed analysis of Lee's results is carried out, small differences with our model appear: we performed numerical simulations using the same assumptions as Lee, except that we used our local optimization procedure instead of the percolation method. We noted two differences:

a) first, the percolation method gives values of $\overline{\ln \mathcal{R}}$ slightly lower than those predicted by equation $(3 \mathrm{a})\left({ }^{4}\right)$. However, this difference goes to zero when $N$ increases: for the lowest temperature and the shortest system $(N=12)$ the difference is of $20 \%$, whereas for the highest temperature and the longest system $(N=143)$ it is only $12 \%$;

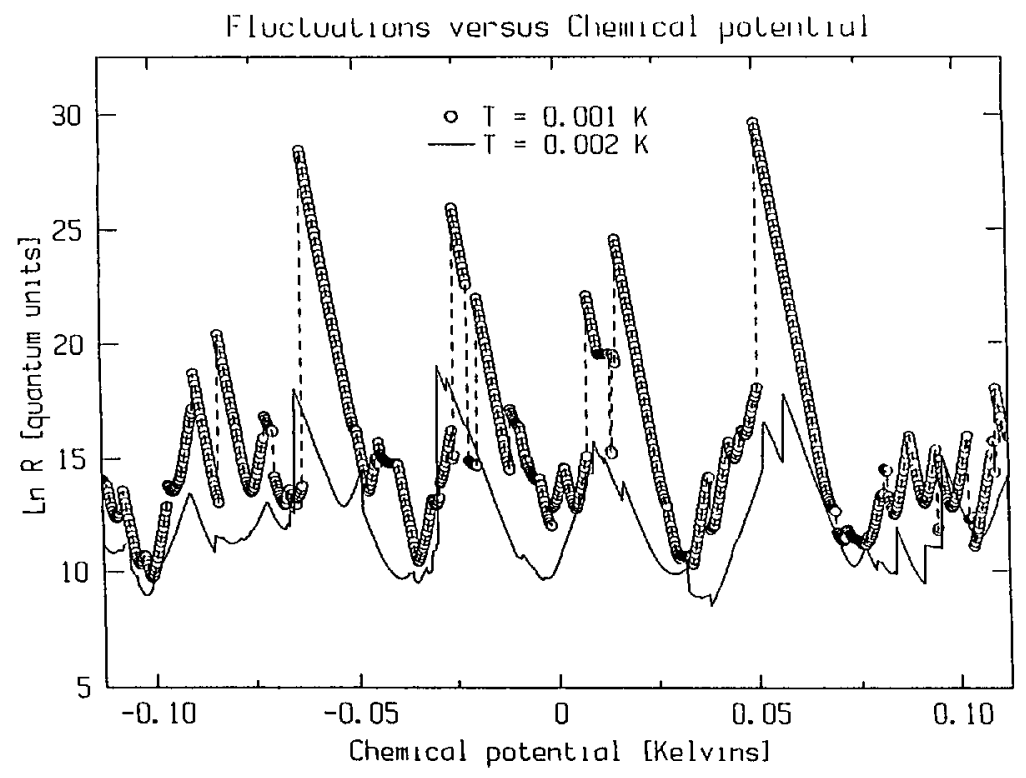

Fig. 3. - Simulation of geometrical fluctuations versus Fermi energy, using the same parameters as in [9]: $L / \xi=1000, \xi=50 l$, density of states $=1$ per Kelvin. Comparison with [9] reveals differences between the shape of fluctuations obtained by the percolation method and by local optimization. Note that the vertical slopes - which are responsible for the crossings of curves at different temperatures - are artefacts of the local optimization procedure.

b) the second point is that the conductance fluctuations (see Fig. 3) as the Fermi energy is varied are often not symmetric. Indeed, as shown in figure 3, one can see that some fluctuations have vertical slopes on one side. We checked that at these points the local optimization

\footnotetext{
$\left({ }^{4}\right)$ To make this comparison, due to differences of notation in [9], one has to take: $T_{0}=0.04$ and $r_{0} \equiv \frac{n_{0} \xi}{4}$
} 
procedure fails, i.e. the optimal path cannot be obtained by optimizing at each "step". Nevertheless, this does not change much the overall size of the fluctuations: for $N$ of order 50 , the difference between the results of reference [9] and equation (3b) is less than $10 \%$, and goes to zero as $N$ increases.

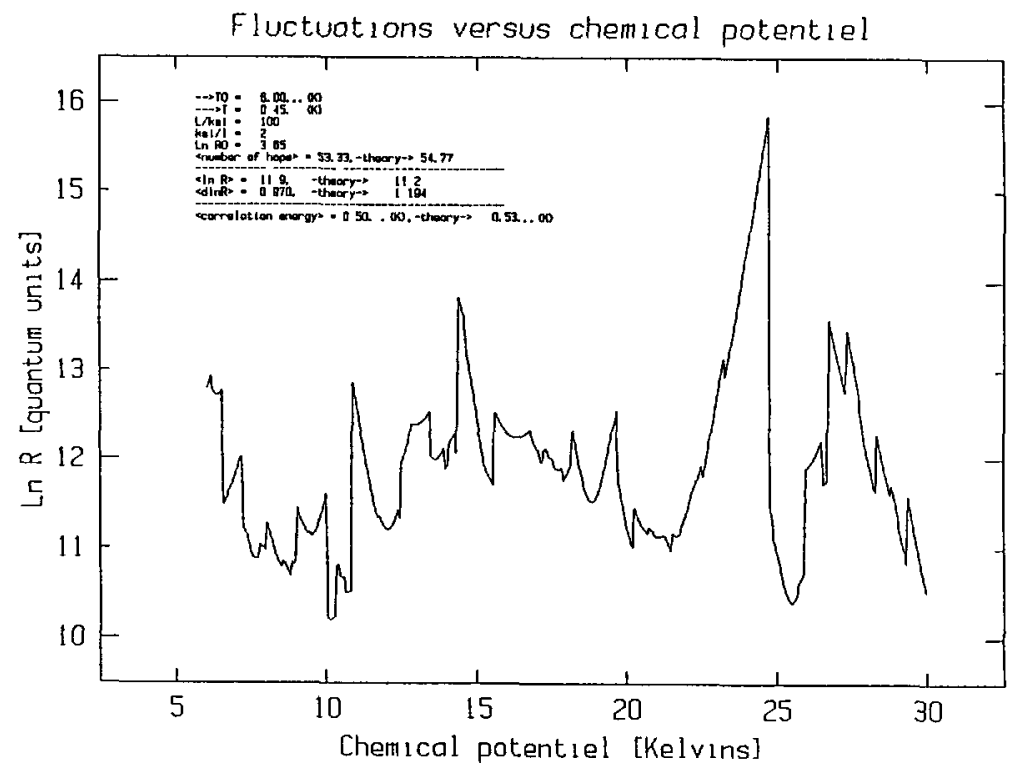

Fig. 4. - Simulation of the wire of [3] with our model. $L / \xi=100, \xi=2 l, T_{0}=6.0 \mathrm{~K}$. Predictions of our theoretical model are in good agreement with numerical results. "<Correlation energy $>$ " means the average energy width of fluctuations.

Therefore, we conclude that our assumption of local optimization is only exact asymptotically but gives quite good predictions for the sizes we are interested in. For the sample studied in reference [3], and in the regime where our model holds, $\xi$ is estimated to be of the order of, or slightly larger than, $l$, the distance between impurities. This contrasts strongly with Lee's simulations where $\xi$ extends over 50 localized sites. Since $T_{0} \sim \xi^{-1}$, we expect much larger values of $T_{0}$ for this sample than in reference [9] $\left(^{5}\right)$. Figure 4 shows the result of a simulation adapted to sample of reference [3]: $\frac{L}{\xi}=100, \xi=2 l$. Experimental values of $\ln \mathcal{R}$ are typically $\sim 9$ at $T=0.45 \mathrm{~K}$ (see the most resistive set of curves of Fig. 4 in Ref. [3]). In order to take into account the fact that our local optimisation method overestimates $\ln \mathcal{R}$ by $\sim 20 \%$, we adjusted $T_{0}$ value so that $\ln \mathcal{R}$ (as given by (3a)) $\simeq 11.5$ at $T=0.45 \mathrm{~K}$ and found $T_{0}=6.0 \mathrm{~K}$. $T_{0}$ is found to vary between $2 \mathrm{~K}$ and $10 \mathrm{~K}$ from the barely insulating to the strongest insulating regime observed.

We shall now discuss in more detail the nature of these fluctuations for a given sample, as the temperature or the gate voltage (i.e. the Fermi level) is varied.

i) Temperature. The main point of the above analysis is that the resistance of the sample is entirely governed by a certain pair of impurities, between which transport is most difficult

\footnotetext{
$\left({ }^{5}\right)$ As a consequence, the temperature range where Variable Range Hopping holds should also be much larger than in reference [9].
} 


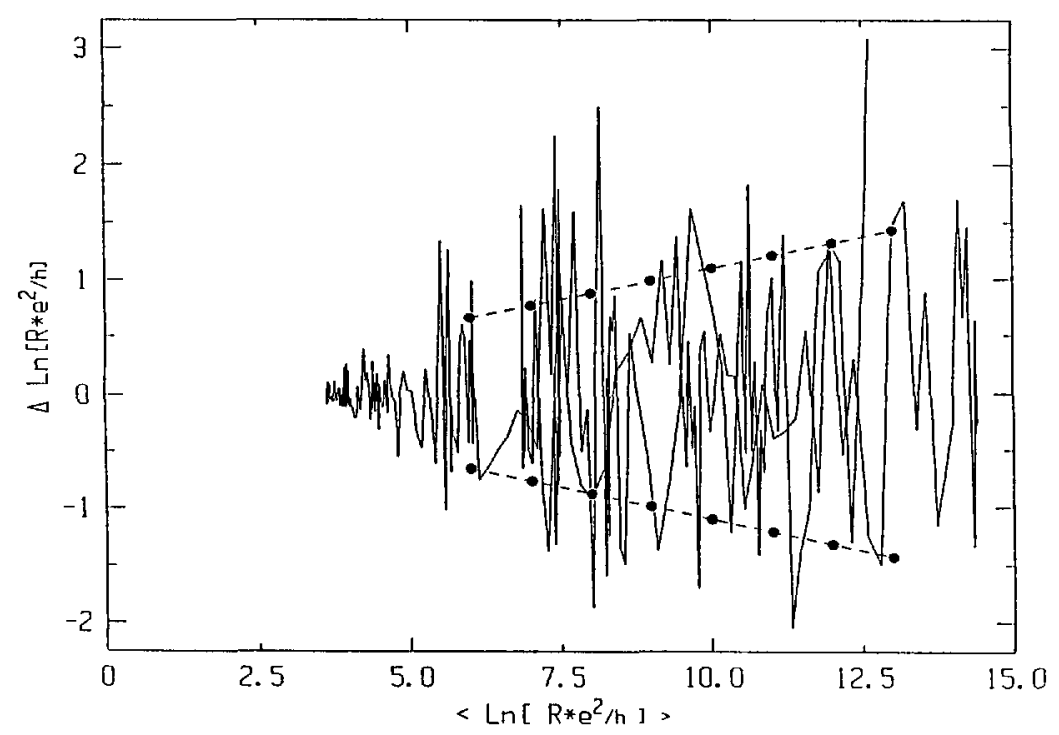

Fig. 5. $-\ln \mathcal{R}$ versus $\overline{\ln \mathcal{R}}$ as experimentally determined (see [3]); the dotted line shows our prediction for the typical fluctuations.

$[9,12]$. Crudely speaking, the large resistance arises either because the distance between the sites is large $\simeq n_{\max } \xi$, but their energies are rather small (Type I), or because they are close in space but require a large energy $\simeq n_{\max } k T$ from the thermal bath (Type II). Suppose at a given temperature $T_{1}$, the weak link is of type II. Its resistance thus quickly increases as $T$ decreases. It becomes more and more probable that this link will be "shunted" by a longer but less energetic type I link. It is relatively easy to show, using excluded area arguments much as above, that this will occur at a temperature $T_{2}$ such that, typically, $\frac{\left(T_{1}-T_{2}\right)}{T_{2}} \simeq \frac{1}{\ln (a N)}$.

Conversely, a low energy type I link will soon loose its "weak link status" as the second largest resistance grows. The temperature at which the switch occurs is given by the same expression. The temperature dependence of a given sample is summarized in figure 2: at low temperatures, it is given by a succession of plateaus and activated regions, oscillating around the mean (Mott) behaviour $n_{0} \sqrt{2 \ln (a N)}$. The size of these regions is roughly constant in $\ln$ scale, and goes to zero with the length of the sample (as $\left.\frac{1}{\ln (a N)}\right)$.

ii) Gate Voltage. Following the discussion of Lee [9] (see also [12]), one sees from equation (1) that as the Fermi energy $E_{\mathrm{F}}$ is varied, two cases may occur: either both sites are on the same side of the Fermi level, and $\ln \mathcal{R}$ varies as $\pm \frac{E_{\mathrm{F}}}{k T}$, or they are on opposite sides and $\ln \mathcal{R}$ is constant. Since the fluctuations of $\ln \mathcal{R}$ are of order $\frac{n_{0}^{2}}{n_{\max }}$ and assuming that varying $E_{\mathrm{F}}$ is tantamount to changing the disorder, the peak-to-peak distance $\Delta E_{\mathrm{f}}$ is found to be of the order of $\frac{n_{0}^{2} / n_{\max }}{1 / 2 k T} \simeq \frac{2 n_{0} k T}{\sqrt{2 \ln a N}} \simeq 0.5-1.0 \mathrm{~K}$ for the experimental data. This value is, in fact, roughly the mean level spacing in energy within a box of length $r_{\max }$. But, as very recent simulations at $T=0 \mathrm{~K}[17]$ showed, the typical width in energy of quantum fluctuations is given by the mean level spacing within the considered coherent system: intuitively, the transmission changes abruptly around every resonance. Assuming, as usual, that quantum coherence is preserved 
on the scale of each hop, we find that quantum fluctuations within the dominant link must be taken into account since their typical energy scale is also the mean level spacing within the box of length $r_{\max }$. Indeed, since the resistance of a given sample is that of the most resistive link, quantum fluctuations could only be neglected if they varied much more slowly with energy than the "geometrical" fluctuations, which, typically, is not true. Of course, these arguments deal only with average energy scales. Therefore, the precise origin (geometrical or quantum) of fluctuations versus Fermi energy will depend on each particular case. Experimentally, if an observed fluctuation is strongly temperature dependent, one will say that this fluctuation is of geometrical origin. Indeed, if a fluctuation is only due to moving Fermi energy in equation (1), one sees easily that shifting temperature will change it strongly (see Fig. 1 of [9], and Fig. 3 ). On the contrary, a fluctuation of quantum origin will be temperature independent, in the whole range of temperatures where the dominant link remains the same [3].

Let us now consider the case of a fluctuation purely of quantum origin. One should realize that this fluctuation cannot develop fully, for reasons similar to those mentioned above: imagine that as the Fermi energy is varied, one encounters a resonance which considerably enhances the transmission coefficient across the weak link. In this case, this link will simply disappear from the game, and the second largest resistance will become the largest (the probability for simultaneous resonances being negligible). One can shown that the difference between the $\ln$ of the largest and the second largest resistance is also of order $\frac{n_{0}^{2}}{n_{\max }}$ Similarly, if interference effects cause the transmission across the "weak link" to be considerably less than its most probable value, a better site will be found, again limiting the drop to $\frac{n_{0}^{2}}{n_{\max }}$ We thus conclude that one directly observes (due to the dominance of a single link) quantum fluctuations only when they are smaller than $\frac{n_{0}^{2}}{n_{\max }}$ For larger fluctuations, these quantum fluctuations are self consistently truncated because of the presence of the other links. In particular, contrarily to the ideas of [4], one never observes true resonances except at very low temperatures or for short samples (see below). The latter point was first established in [11].

We have thus reached the conclusion that "truncated quantum fluctuations" occur when $w_{\text {geo }} \gg w_{q}>\frac{n_{0}^{2}}{n_{\max }}$ As mentioned above, one may estimate quantum fluctuations as : $w_{\mathrm{q}}=$ $(\Delta \ln \mathcal{R})_{\text {Quant. }} \simeq C_{\omega}\left(\frac{r}{\xi}\right)^{\omega} \simeq n_{\max }^{\omega}$, with $\omega=1 / 2$ in the barely localised regime [5] or $\omega \sim 1 / 5$ in the directed path regime. Comparing with $\frac{n_{0}^{2}}{n_{\max }}$, one finds that quantum fluctuations are truncated for long samples or sufficiently high temperatures, more precisely when $\ln (a N)>$ $n_{0}^{\frac{1-\omega}{1+\omega}}$. (For simplicity, we set $C_{\omega}=1$ ). Note that, since the dominant link is characterized by the absence of energy levels around the energy of the electron, one could ask whether the results of [5] are directly relevant to our case or not. A specific numerical study of this point would be interesting: one could expect that the localization length and the fluctuations are somewhat reduced compared to their typical values (i.e. without any restrictions on the energy levels). However, quantum fluctuations cannot be smaller than those predicted by a directed path approximation [6]. Indeed, the whole justification of this model is the very absence of nearby resonances between initial and final sites and one of the results is that amplitude of fuctuations is independent of the separation $W$ in energy between the electron energy and intermediate levels. Though interesting, a precise calculation of the amplitude of the quantum fluctuations within the dominant link is not required here, since for both $\omega=1 / 5$ or $1 / 2$ we find that quantum fluctuations are truncated in our experiments (except perhaps for the 
lowest temperatures) and we expect equation ( $3 \mathrm{~b})$ to hold. This prediction is compared with the experiments in figure 5 , where $\ln \mathcal{R}$ is plotted versus the smoothed $\overline{\ln \mathcal{R}}$ (we took $2 \ln (a N)=9$ ). Agreement is reasonably good. Note in particular that the experimental sample is longer than those used in the numerical simulations: this is why (see Eq. (3b)) the observed fluctuations are substantially smaller than those reported by Lee (Fig. 1 of Ref. [9]). Finally, the fluctuations seem to depart somewhat from equation $(3 b)$ for the highest resistances. This may be a sign that one enters the purely quantum regime, with a small value of $\omega$.

In conclusion, we have shown, through a detailed analysis of the Mott conduction mechanism in one dimension, that the large conductance fluctuations in disordered insulators result from a subtle interplay between purely quantum phenomena and geometrical fluctuations arising from the energies and locations of the impurities. Our results are in agreement with previous investigations and are successfully compared to both experiments (see the companion paper) and numerical simulations.

\section{Acknowledgements.}

We want to thank J.L. Pichard for many theoretical discussions and M. Sanquer for having performed the experiments motivating this work and for constant collaboration. Finally, we thank Pr. B. Shklovskii for drawing our attention on reference [12], which, as we discovered, contains many of the ideas expressed here.

\section{References}

[1] Mesoscopic Phenomena in Solids, B.L. Altshuler, P.A. Lee and R.A. Webb Eds. (Elsevier Science Publisher, 1991).

[2] Fowler A.B., Hartstein A., Webb R.A., Phys. Rev. Lett. 48 (1982) 196;

Laiko E.I., Orlov A.O., Savchenko A.K., Ilyichev E.A., Poltoratsky E.A. Zh Eksp Teor Fiz. 93 (1987) 2204 (Sov. Phys. JETP 66 (1987) 1264).

[3] Ladieu F., Mailly D., Sanquer M., J. Phys. I France 3 (1993).

[4] Azbel M.Y., Hartstein A., DiVincenzo D.P., Phys. Rev. Lett. 52 (1984) 1641;

Azbel M. Y., Solid State Commu. 45 (1983) 527.

[5] Pichard J.L., Zanon N., Imry Y., Stone A.D., J. Phys. France 51 (1990) 587;

Pichard J.L., Quantum coherence in mesoscopic systems, B. Kramer Ed., NATO Advanced Studies Institute Series (Plenum, New York, 1990).

[6] Kardar M., Medina E., Shapir Y., Wang W.S., Phys. Rev. Lett. 62 (1989) 941 and 64 (1990) 1816;

Medina E., PhD Dissertation (June 1991) (MIT), unpublished.

[7] Mott N.F., Philos. Mag. 26 (1972) 1015.

[8] Mott N.F., J. Non-Cryst. Solids 1 (1968) 1.

[9] Lee P.A., Phys. Rev. Lett. 53 (1984) 2042.

[10] Serota R.A., Kalia R.K., Lee P.A., Phys. Rev. B 33 (1986) 8441.

[11] Stone A.D. and Lee P. Phys. Rev. Lett. 54 (1985) 1196.

[12] Raikh M.E, Ruzin I.M., Sov. Phys. JETP 68 (1989) 642;

See also Raikh M.E., Ruzin I.M., [1] p. 315.

[13] Ambegaokar V., Halperin B., Langer J.S., Phys. Rev. B4 (1971) 2612.

[14] Sivan U., Imry Y., Phys. Rev. B 35 (1987) 6074.

[15] Kurkijarvi J., Phys. Rev. B 8 (1973) 922.

[16] see, e.g. Bouchaud J.P., Georges A., Phys. Rep. 195 (1990) 127 and Appendix 1 of J. Phys. II France 1 (1991) 1465.

[17] Avishai Y., Pichard J.-L., Muttalib K.A., J. Phys. I. France 3 (1993). 